\title{
RESEARCH
}

Open Access

\section{Early functional outcome after IV rTPA administration in Egyptian acute ischemic stroke patients}

\author{
Mohamed A. Elsayed, Husam Salah, Asmaa Sabbah* (10, Ghada Hatem and Mona K. Moawad
}

\begin{abstract}
Background: Intravenous thrombolysis has become a standard practice in eligible patients with acute ischemic stroke (AIS). Functional outcome after AIS is a major concern for patients, families, and neurologists. Identifying factors affecting the early functional outcome and the effect of IV rTPA in Egyptian AIS patients is crucial to address concerns and queries.

Objectives: Compare functional outcome between AIS patients treated with and without IV rTPA and to study clinical predictors of outcome.

Methods: This study was performed on AIS within $24 \mathrm{~h}$ of symptom onset. Patients were dichotomized into group I, patients who received IV rTPA, and group II, patients who had contraindication to it. Patients were assessed with NIHSS and mRS at 3 months. The functional outcome was studied in relation to risk factors, TOAST classification, OSCP classification, and NIHSS severity.

Results: In mild stroke: group I showed better 7-day NIHSS and 3-month mRs, when compared to group II, (P 0.002, 0.089 respectively). In group I, $68.6 \%$ of patients with total anterior circulation syndrome (TACS) had unfavorable outcome $(P=0.004)$. Those with lacunar syndrome, $78.6 \%$ had favorable outcome $(P=0.0162)$. In group I, there was a positive correlation between 3-month mRs and age, NIHSS on admission, and NIHSS at 7 days $(P 0.017,<$ $0.001,<0.001$, respectively).

Conclusion: Age and NIHSS at admission were correlated with poor outcome at 3 months in the IV rTPA group. Better functional outcome was observed in thrombolysed patients in groups with mild stroke severity. The study showed that patients with TACS had the most unfavorable outcome, while lacunar infarction patients had the most favorable outcome.
\end{abstract}

Keywords: Acute ischemic stroke; Outcome; Thrombolysis; IV rtPA; Egyptian

\section{Introduction}

Intravenous thrombolysis has become a standard practice in eligible patients with acute ischemic stroke. According to the National Institute of Neurological Disorders and Stroke (NINDS) trial, patients who were treated with recombinant tissue plasminogen activator (rTPA) were at least 30\% more likely to have a favorable outcome (modified Rankin Scale 0-1) at three months, when compared to patients who received placebo [1]. Functional outcome after acute ischemic stroke is a major concern for patients,

* Correspondence: doctor_sabbah@cu.edu.eg

Neurology Department, Kasr Alainy school of medicine, Cairo University, Cairo, Egypt

Springer Open families, and neurologists. Identifying factors affecting the early functional outcome is crucial to address concerns and queries.

The aim of the study was to define effect of thrombolysis and other factors on early functional outcome in an Egyptian cohort of acute ischemic stroke patients who were treated with IV rTPA and compare them to patients who had one or more contraindications to IV rTPA administration. We also tried to explore the possible difference in functional outcome after thrombolysis in different stroke subtypes, based on etiological classifications and vascular territorial distribution. 


\section{Methods}

This study was carried out in the stroke unit, Neurology department, Cairo University, in the period from May 2017 to October 2017. Patients were eligible for inclusion in the study if they were $\geq 18$ years, had received the clinical diagnosis of acute ischemic stroke, and presenting within $24 \mathrm{~h}$ of symptom onset. Included patients were dichotomized into 2 groups: group I included patients who were eligible for thrombolysis and received IV rTPA and group II included patients who presented out of window or had one or more contraindication to IV rTPA administration. IV rTPA was administered according to the American Heart Association/American Stroke Association AHA/ASA guidelines for early management of acute ischemic stroke 2015 scientific statement [2].

The IV thrombolysis administered was alteplase (Actilyse) by Boehringer-Ingelheim, Germany.

Exclusion criteria were applied to the two groups for better matching between them.

Exclusion criteria: patients were excluded from the study if there was any past history of intracranial hemorrhage and known arteriovenous malformation, neoplasm, or aneurysm. They were also excluded if there was history of ischemic stroke or central nervous system surgery within the previous 3 months of stroke onset, gastrointestinal or urinary tract hemorrhage within 3 weeks of stroke onset or current use of an anticoagulant, and if the onset of stroke symptoms started beyond $24 \mathrm{~h}$. According to the non-contrast CT scan of the brain, patients were excluded if there was evidence of intracranial hemorrhage or an established hypodensity occupying more than one-third of a hemisphere. Other exclusion criteria are if the blood pressure remained uncontrolled, systolic blood pressure $>185 \mathrm{~mm} \mathrm{Hg}$ or diastolic blood pressure remains $>110 \mathrm{~mm} \mathrm{Hg}$ despite repeated measurements, severe stroke as objectively measured by NIHSS $>25$, glucose less than $50 \mathrm{mg} / \mathrm{dl}$ or more than $400 \mathrm{mg} / \mathrm{dl}$, and platelet count below normal limit.

Patients were assessed clinically, at the time of presentation to the emergency department (ED) after stroke onset and at 1, 2, 24 h, and on days 7, 30, and 90. They were subjected to general, neurological examination and monitoring of vital signs.

The National Institute of Health Stroke Scale (NIHSS), a 15-item scale that measures the level of neurologic impairment. Total scores on the NIHSS range from 0 to 42 , with higher values indicating more severe neurologic impairment [3]. According to the NIHSS, strokes were categorized into mild NIHSS $(\leq 8)$, moderate NIHSS [4-10], and severe stroke NIHSS $(\geq 16)$. Included patients were assessed with NIHSS on admission and on day 7 .

Clinical outcome of patients was assessed by the modified Rankin Scale (mRs) at 3 months. The modified Rankin Scale, a measure of disability. Scores on the modified Rankin Scale range from 0 (no symptoms at all) to 6 (death).
Patients clinical outcome was categorized as favorable (score 0-2) or unfavorable (score 3-6) [11].

Patients were categorized according to stroke etiology based on Toast classification into 4 stroke categories: large-artery atherosclerosis, cardio-embolism, small-vessel occlusion (lacune), and "others" which included stroke of other determined etiology and stroke of undetermined etiology [12]. Based on the vascular territory distribution of stroke in brain imaging, patients were classified according to The Oxfordshire Community Stroke Project (OCSP) clinical classification of subtypes of cerebral infarction. OCSP includes total and partial anterior circulation infarction, lacunar infarction, and posterior circulation infarction. OCSP can be used to predict early mortality, functional outcome, and whether the infarct was due to a large- or small-vessel occlusion. The OCSP classification was originally developed and tested by neurologists as part of a community-based study of first-ever stroke [13].

The following laboratory investigations were performed: complete blood count, coagulation profile, blood sugar, renal functions, and electrolytes. Radiological investigations: non-contrast computed tomography (CT): it was done on a GE bright speed 16 scanner at the computed tomography unit, Emergency Department, Kasr AL Ainy Hospital, that is available $24 \mathrm{~h}$ every day. The main role of $\mathrm{CT}$ is to exclude intracerebral hemorrhage and stroke mimics. Magnetic resonance imaging (MRI): was not routinely done to all patients, and done only in cases where there was suspicion for the diagnosis of ischemic infarction, or in patients showing deterioration of the clinical condition without evident cause on CT brain. It was performed on a 1.5-T Phillips Intera scanner at the Magnetic Resonance Unit, Radiology Department, Kasr AL Ainy Hospital. The following protocols were used: T1-weighted images (axial), T2-weighted images (axial), fluid-attenuated inversion recovery (FLAIR) (axial), diffusion-weighted images (DWI), and apparent diffusion coefficient (ADC). DWI and corresponding ADC maps are the most sensitive imaging modality in detection of brain infarction. It can detect infarction within minutes from onset. Its sensitivity is up to $73-92 \%$ in the first $3 \mathrm{~h}$ and up to $95-100 \%$ in the first $6 \mathrm{~h}$ [14]. All patients did the rest of the battery of investigations routinely done in the stroke unit, Neurology department, Cairo University, including extracranial and transcranial carotid and vertebro-basilar duplex added to echocardiography.

\section{Statistical methods}

Data were coded and entered with SPSS (Statistical Package for the Social Sciences) version 25, 2017. Mean, standard deviation, median, minimum, and maximum were used in quantitative data. Frequency and relative frequency were used for categorical data. Comparisons were done using the non-parametric Kruskal-Wallis and 
Mann-Whitney tests. The chi-square test was used for comparisons between categorical data. Correlations between quantitative variables were done by Spearman's correlation coefficient. $P$ values less than 0.05 were considered statistically significant.

\section{Results}

Patients in this study were distributed into 2 groups: group I, who received IV rTPA that included 75 patients, and group II, who did not receive IV rTPA and presented within the first $24 \mathrm{~h}$ after stroke onset. Group II included 114 patients. Demographics, baseline characteristics, and NIHSS severity classification in both groups are illustrated in Table 1.

\section{Comparisons of functional outcome in both groups}

In group $\mathrm{I}, \mathrm{mRs}$ at 3 months from onset ranged between 0 and 6 , with a median of 2 . In group II, it ranged between 0 and 6 , with a median of 2 . There was no statistically significant difference between both groups $(P=$
0.3516). In group I, at 3 months from onset, 39 patients (52\%) had favorable outcome, and 36 patients (48\%) had unfavorable outcome, while in group II, 55 patients (48\%) had favorable outcome, and 59 patients (52\%) had unfavorable outcome. There was no statistically significant difference between both groups $(P=0.5915)$.

\section{Comparisons of functional outcome in group I and group II subtypes based on NIHSS severity}

In mild stroke: group I showed better mean rank at 7 days NIHSS, 7 days mRS, and 3 months mRs, when compared to group II; this difference was statistically significant $(P 0.002,0.016$, and 0.089 , respectively) (Table 2$)$; 3 -month mRs in mild, moderate, and severe strokes in both groups is illustrated in Figs. 1, 2, and 3.

\section{Clinical outcome within group I (subgroup analysis)}

Based on TOAST classification: patients with large vessel disease, 7 patients (46.7\%) had favorable outcome, and 8 patients (53.3\%) had unfavorable outcome, with no statistically

Table 1 Demographic and baseline characteristics of both groups

\begin{tabular}{|c|c|c|c|c|c|c|c|}
\hline & & \multicolumn{2}{|l|}{ Group I } & \multicolumn{2}{|l|}{ Group ॥ } & \multirow[t]{2}{*}{$x^{2}$ value } & \multirow[t]{2}{*}{$P$ value } \\
\hline & & $\bar{N}$ & $\%$ & $\bar{N}$ & $\%$ & & \\
\hline Age & & $60.24 \pm 9.37$ & & $58.81 \pm 13.31$ & & & 0.37 \\
\hline Male & & 51 & 68.0 & 62 & 54.4 & 3.487 & 0.062 \\
\hline Hypertension & & 49 & 65.3 & 62 & 54.4 & 2.237 & 0.135 \\
\hline \multirow[t]{3}{*}{ Cardiac diseases } & $\mid H D^{\$}$ & 24 & 32 & 31 & 27.2 & 0.507 & 0.477 \\
\hline & $\mathrm{RHD}^{+}$ & 5 & 6.7 & 7 & 6.1 & 0.021 & 1 \\
\hline & $\mathrm{AF}^{\ddagger}$ & 12 & 16 & 28 & 24.6 & $1.987^{\mathrm{a}}$ & 0.159 \\
\hline Smoker & & 24 & 32 & 27 & 23.7 & 1.588 & 0.208 \\
\hline \multirow[t]{3}{*}{ Cerebrovascular ischemic event } & Stroke & 12 & 16 & 25 & 21.9 & 2.636 & 0.450 \\
\hline & TIA & 5 & 6.7 & 3 & 2.6 & & \\
\hline & TIA + stroke & 1 & 1.3 & 2 & 1.8 & & \\
\hline Diabetes Mellitus & & 27 & 36 & 40 & 35.1 & 0.016 & 0.898 \\
\hline Hyperlipidemia & & 28 & 37.3 & 35 & 30.7 & 0.882 & 0.348 \\
\hline \multirow[t]{3}{*}{ Stroke Severity } & Mild (NIHSS" 0-8) & 21 & 28.0 & 43 & 37.7 & 1.920 & 0.383 \\
\hline & Moderate (NIHSS"l 9-15) & 37 & 49.3 & 48 & 42.1 & & \\
\hline & Severe (NIHSS" $\geq 16$ ) & 17 & 22.7 & 23 & 20.2 & & \\
\hline \multirow[t]{4}{*}{ TOAST classification } & Large vessel & 15 & 20.0 & 20 & 17.5 & 0.187 & 0.6658 \\
\hline & Cardioembolic & 24 & 32.0 & 44 & 38.6 & 0.851 & 0.3563 \\
\hline & small vessel & 12 & 16.0 & 15 & 13.2 & 0.288 & 0.5917 \\
\hline & Others & 24 & 32.0 & 35 & 30.7 & 0.035 & 0.8507 \\
\hline \multirow[t]{4}{*}{ OCSP" classification } & $\operatorname{TACS}^{* *}$ & 35 & 46.7 & 34 & 29.8 & 5.545 & $0.0185^{*}$ \\
\hline & $\mathrm{PACS}^{+\dagger}$ & 24 & 32.0 & 60 & 52.6 & 7.734 & $0.0054^{*}$ \\
\hline & LACS $^{\neq \neq}$ & 14 & 18.7 & 15 & 13.2 & 1.046 & 0.3065 \\
\hline & $\mathrm{POCS}^{\S \S}$ & 2 & 2.7 & 5 & 4.4 & 0.363 & 0.5471 \\
\hline
\end{tabular}

${ }^{*} P$ value ${ }^{<} 0.05$ is significant, ${ }^{\$}$ ischemic heart disease, ${ }^{\dagger}$ rheumatic heart disease, ${ }^{\ddagger}$ atrial fibrillation, ${ }^{\S}$ transient ischemic attack, ${ }$ National institute of health,

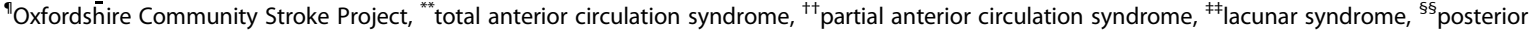
circulation syndrome 
significant difference $(P=0.72)$. Patients with cardioembolic etiology, 12 patients $(50 \%)$ had favorable outcome, and 12 patients (50\%) had unfavorable outcome; with no statistically significant difference $(P=1)$. Patients with small vessel disease, 8 patients $(66.7 \%)$ had favorable outcome, and 4 patients (33.3\%) had unfavorable outcome, with no statistically significant difference $(P=0.1)$. Patients with other etiologies: 12 patients $(50 \%)$ had favorable outcome, and 12 patients (50\%) had unfavorable outcome; with no statistically significant difference, $(P=1)$. So, etiological subtypes (according to TOAST classification) did not have an impact on the outcome of patients receiving rTPA, as shown in Table 3.

Based on OCSP classification: patients with total anterior circulation syndrome: 11 patients (31.4\%) had favorable outcome and 24 patients (68.6\%) had unfavorable outcome with significant difference $(P=0.0044)$, while those with partial anterior circulation syndrome: 16 patients $(66.7 \%)$ had favorable outcome and 8 patients (33.3\%) had unfavorable outcome with significant difference $(P=0.0451)$. And of those with lacunar syndrome: 11 patients $(78.6 \%)$ with had favorable outcome and 3 patients $(21.4 \%)$ had unfavorable outcome with significant difference $(P=0.0162)$. Patients with posterior circulation syndrome were only 2 patients, as shown in Table 4 .

Based on onset of symptoms to treatment (time to treatment): group I was further subdivided into those received IV rTPA within $\leq 3 \mathrm{~h}$ (group Ia) and those received IV rTPA within 3-4.5 h (group Ib). There was no statistically significant difference between both groups regarding outcome, $(P=0.588,0.924)$.

\section{Correlations in group I}

In group I, there was statistically significant positive correlation between 3-month mRs, on one hand, and age, NIHSS on admission, and NIHSS at 7 days, on the other hand, which means that worse outcome as assessed by mRs was directly proportionate to age, NIHSS on admission, and

Table 2 Comparisons of 7-day NIHSS, 7-day mRS, and 3-month mRs in both groups' subtypes based on NIHSS severity

\begin{tabular}{|c|c|c|c|c|}
\hline \multirow[t]{2}{*}{ Stroke severity } & \multirow[t]{2}{*}{ NIHSS"I } & \multirow{2}{*}{$\begin{array}{l}\text { Group I } \\
\text { Mean rank }\end{array}$} & \multirow{2}{*}{$\begin{array}{l}\text { Group II } \\
\text { Mean rank }\end{array}$} & \multirow{2}{*}{$\begin{array}{l}P \\
\text { value }\end{array}$} \\
\hline & & & & \\
\hline \multirow[t]{3}{*}{ Mild stroke } & 7-day NIHSSII & 21.15 & 36.43 & 0.002 \\
\hline & 7-day $\mathrm{mRS}^{+}$ & 24.35 & 35.56 & 0.016 \\
\hline & 3-month $\mathrm{mRs}^{\dagger}$ & 25.23 & 34.49 & 0.089 \\
\hline \multirow[t]{3}{*}{ Moderate stroke } & 7-day NIHSS" & 36.36 & 43.05 & 0.194 \\
\hline & 7-day $\mathrm{mRS}^{+}$ & 38.62 & 46.38 & 0.137 \\
\hline & 3-month $\mathrm{mRs}^{\dagger}$ & 41.18 & 39.01 & 0.853 \\
\hline \multirow[t]{3}{*}{ Severe stroke } & 7-day NIHSSII & 13.31 & 18.68 & 0.117 \\
\hline & 7-day $\mathrm{mRS}^{+}$ & 19.32 & 21.37 & 0.58 \\
\hline & 3-month $\mathrm{mRs}^{\dagger}$ & 13.73 & 18.39 & 0.481 \\
\hline
\end{tabular}

${ }^{*} P$ value ${ }^{<} 0.05$ is significant, "National Institute of Health Stroke Scale, ${ }^{\dagger}$ modified Rankin scale
NIHSS at 7 days $(r=0.286,0.529,0.72)(P=0.017,0.001$, $0.001)$.

\section{Discussion}

This study aimed at evaluating the impact of IV rTPA on early outcome in acute ischemic stroke patients and clarifies the factors that could affect this outcome. Accordingly, 75 patients (Group I) who received rTPA and 114 patients (group II) who did not receive rTPA were recruited from the stroke unit, Neurology department, Cairo University Hospitals, and subjected to neurological evaluation, investigations and follow up at 3 months.

Advanced age is one of the most significant stroke risk factors. Three quarters of all strokes occur in people above age of 65 . The risk of having a stroke doubles each decade after the age of 55 years; mortality risks also increase with age [15]. The present study stated that advanced age correlates significantly with poor outcome at 3 months in acute ischemic stroke patients who received IV rTPA.

Results of this study did not show any significant relation between any of the studied risk factors including sex and outcome after acute ischemic stroke treated with IV thrombolysis. These results agree with Bhardwaj and colleagues, who found that risk factors such as hypertension, diabetes, dyslipidemia, smoking, alcohol intake, history of stroke, coronary artery disease, and valvular heart disease did not influence outcome in patients of acute ischemic stroke receiving rTPA [4].

On the other hand, Spaander and colleagues found that women have a poorer functional outcome after IV rTPA compared with men [5]. Demchuk and colleagues concluded that, no history of diabetes mellitus, normal pretreatment blood glucose level, and normal pretreatment blood pressure were independent predictors of good outcome among patients treated with IV alteplase for acute ischemic stroke [6].

Results of the current study showed that clinical assessment on admission using NIHSS correlated significantly with functional outcome at 3 months in patients with acute ischemic stroke treated with rtPA. So, it is a reliable strong predictor factor for early stroke outcome. This agreed with Bhardwaj and colleagues, who found that higher NIHSS score at the time of onset of stroke is an independent predictor of the poor outcome after thrombolysis with IV alteplase treatment in acute ischemic stroke patients. So, severe strokes on initial presentation (high NIHSS) are associated with worse functional outcome (higher mRS at 3 months) [4]. Also, Vogt and colleagues found that NIHSS early after stroke onset is an important determinant of final stroke outcome in a large heterogeneous patient population [7].

Comparison of functional outcome between both groups, $52 \%$ of group I patients had favorable outcome, 
Independent-Samples Mann-Whitney U Test

NIHSS severity: Mild

group

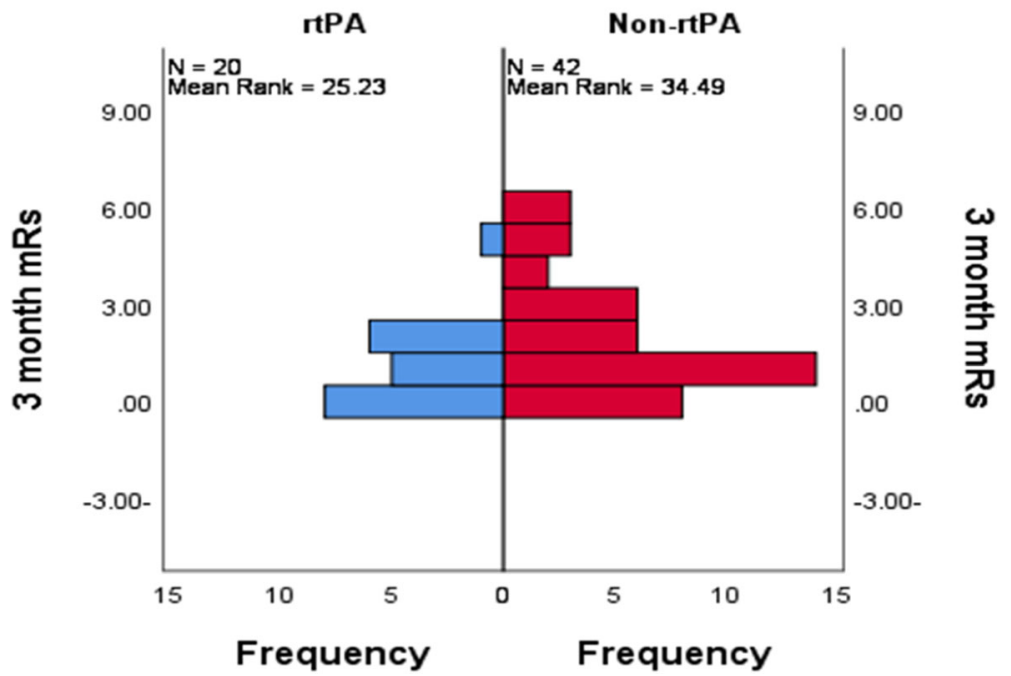

Fig. 1 Modified Rankin scale (mRs) at 3 months in mild stroke in both groups

while $48 \%$ of group II patients had favorable outcome; however, no statistical significance was found $(P=0.59)$. Patients in group I with mild stroke severity showed statistically significant more favorable outcome $(=0.089)$. It could be attributed also to the fact that $46.7 \%$ of patients (35 patients) who received rTPA were TACS in comparison to $29 \%$ (34 patients) in group II, which is associated with poorer outcome.

This current study showed that patients with TACS had the most unfavorable outcome $(P=0.001)$, while lacunar infarction patients had the most favorable outcome $(P=$ $0.001)$. Hence, clinical response to thrombolysis is influenced

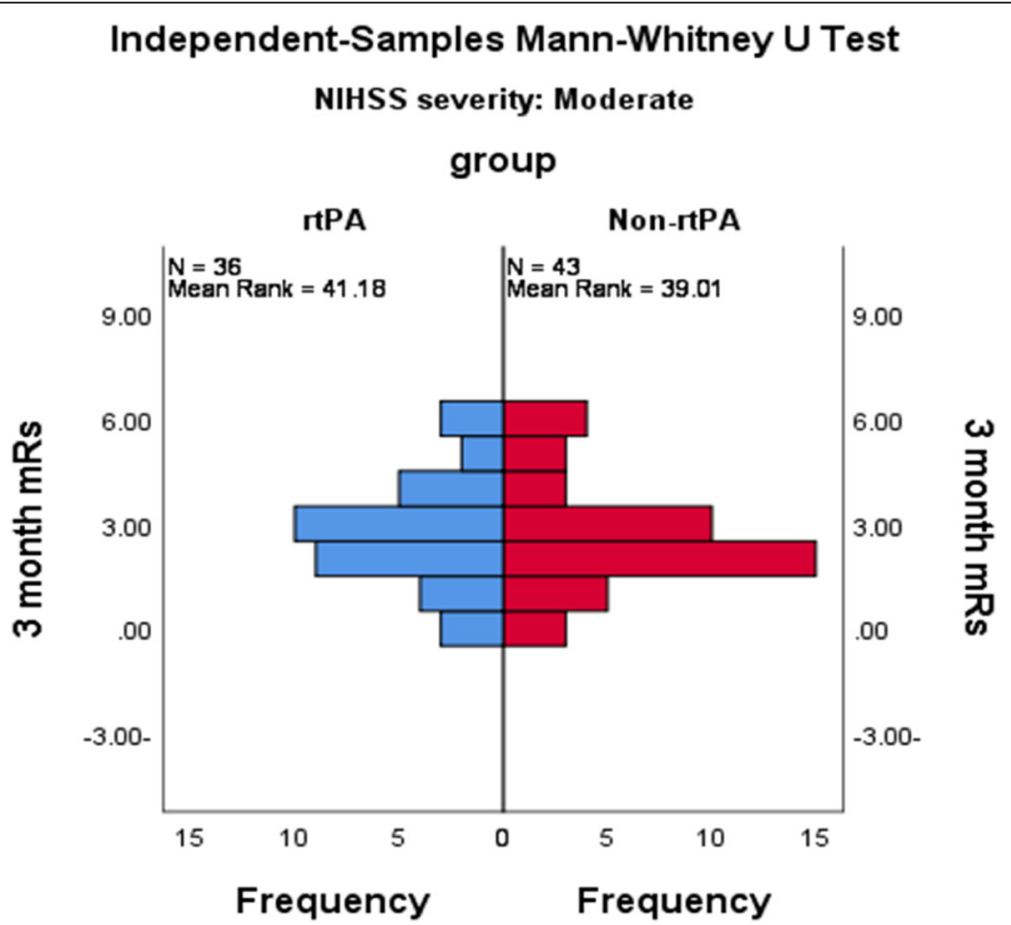

Fig. 2 Modified Rankin scale (mRs) at 3 months in moderate stroke in both groups 
Independent-Samples Mann-Whitney U Test

NIHSS severity: severe

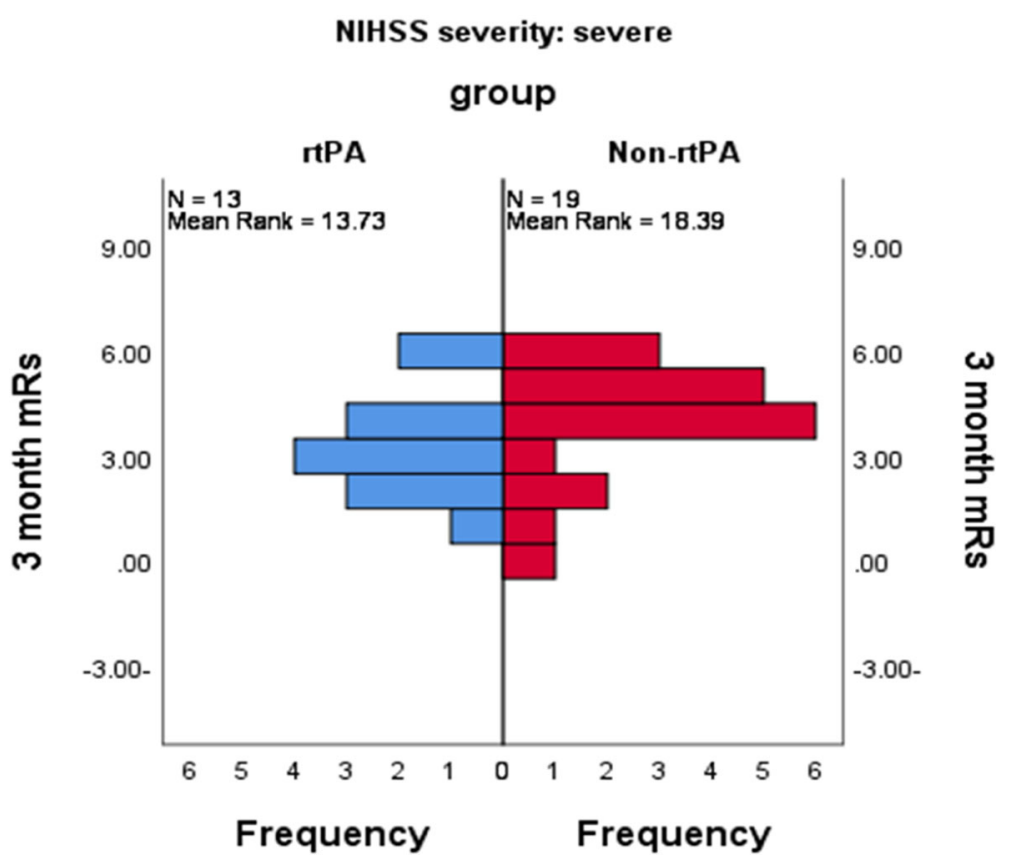

Fig. 3 Modified Rankin scale (mRs) at 3 months in severe stroke in both groups

by the site of occlusion. Yang and colleagues found that after thrombolysis, the patients with TACS exhibited greater spontaneous intracranial hemorrhage (SICH), a higher mortality rate, and worse 3-month clinical outcomes compared with the patients with non-TACS [8].

In group I, $53.3 \%$ of patients with large vessel disease had unfavorable outcome $(P=0.72)$. while only $33.3 \%$ of patients with small vessel disease had unfavorable outcome; $(P=0.1)$. Neither of them was statistically significant. Fuentes and colleagues used multicenter stroke registry data to determine the difference in outcome after thrombolysis in different stroke subtypes: large vessel disease, lacunar, cardioembolic, and other less common types, they found that stroke type did not determine differences in functional outcome at 3 months after IV thrombolysis [9]. Mustanoja and colleagues showed an

Table 3 Outcome of different subtypes of TOAST classification in group I

\begin{tabular}{|c|c|c|c|c|c|c|}
\hline \multirow{2}{*}{$\begin{array}{l}\text { TOAST } \\
\text { classification }\end{array}$} & \multicolumn{2}{|c|}{ Favorable } & \multicolumn{2}{|c|}{ Unfavorable } & \multirow{2}{*}{$\begin{array}{l}X^{2} \\
\text { value }\end{array}$} & \multirow{2}{*}{$\begin{array}{l}P \\
\text { value }\end{array}$} \\
\hline & $N$ & $\%$ & $N$ & $\%$ & & \\
\hline Large vessel & 7 & 46.7 & 8 & 53.3 & 0.126 & 0.7223 \\
\hline Cardioembolic & 12 & 50 & 12 & 50 & 0.000 & 1 \\
\hline Small vessel & 8 & 66.7 & 4 & 33.3 & 2.566 & 0.1092 \\
\hline Others & 12 & 50 & 12 & 50 & 0.000 & 1 \\
\hline
\end{tabular}

${ }^{*} P$ value $<0.05$ is significant independent association of small vessel disease (SVD) and better outcome regardless of stroke severity, and that SVD treated with IV thrombolysis had good outcome than patients with strokes caused by other etiologies [10].

The impact of time-to-treatment on outcome after thrombolysis depends on several factors, including the pattern of arterial occlusion, efficacy of collateral circulation, and size of irreversible ischemia [16]. The results of the present study showed that there was no statistically significant difference in favorable outcome between patients who received rTPA $\leq 3 \mathrm{~h}$ and those who received rTPA at 3-4.5 h and time to treatment correlates significantly with stroke outcome. This could be attributed to the fact that patients presented at a mean time to treatment of $2.67 \mathrm{~h} \pm 0.44$ in the group of patients who received rTPA $\leq 3 \mathrm{~h}$ and no patients were within the first 90 min.

In conclusion The advance in age and admission NIHSS correlated significantly with poor outcome at 3 months in acute ischemic stroke patients who received IV rTPA. Statistically significant better functional outcome was observed in thrombolysed patients than nonthrombolysed, in group with mild stroke severity. Our study showed that patients with TACS had the most unfavorable outcome $(P=0.001)$, while lacunar infarction patients had the most favorable outcome $(P=0.001)$. 
Table 4 Outcome of different subtypes of OCSP classification in group I

\begin{tabular}{|c|c|c|c|c|c|c|}
\hline \multirow[t]{2}{*}{ OCSP Classification" } & \multicolumn{2}{|c|}{ Favorable } & \multicolumn{2}{|c|}{ Unfavorable } & \multirow{2}{*}{$\begin{array}{l}X^{2} \\
\text { value }\end{array}$} & \multirow[t]{2}{*}{$P$ value } \\
\hline & $N$ & $\%$ & $N$ & $\%$ & & \\
\hline Total anterior circulation syndrome (TACS) & 11 & 31.4 & 24 & 68.6 & 9.548 & $0.0044^{*}$ \\
\hline Partial anterior circulation syndrome (PACS) & 16 & 66.7 & 8 & 33.3 & 5.243 & $0.0451^{*}$ \\
\hline Lacunar syndrome (LACS) & 11 & 78.6 & 3 & 21.4 & 24.19 & $0.0162^{*}$ \\
\hline Posterior circulation syndrome (POCS) & 1 & 50 & 1 & 50 & 0 & 0.979 \\
\hline
\end{tabular}

${ }^{*} P$ value ${ }^{<} 0.05$ is significant, ${ }^{\# O x f o r d s h i r e ~ C o m m u n i t y ~ S t r o k e ~ P r o j e c t ~}$

\section{Limitations of the study}

A relatively small number of included patients, as only those who completed the follow-up at 3 months after stroke onset were included; relatively small period of time of patient follow-up (3 months). In our future studies, a larger number of patients shall be included and followed up for longer periods of time.

\section{Acknowledgements}

Not applicable

\section{Informed consent}

Written informed consent was obtained from all participants involved in this investigation prior to the conduct of any study-related activities.

\section{Authors' contributions}

All authors contributed to the research idea and methodology, MK and GH contributed to data collection. MAE and HS analyzed and interpreted the data. AS completed the first draft of the article. All authors contributed to drafting and revising the manuscript. All authors read and approved the final manuscript.

\section{Funding}

No funding sources for this research to be declared (financial or nonfinancial)

\section{Availability of data and materials}

Data supporting the results reported in the article can be retrieved through communication with the corresponding author.

\section{Ethics approval and consent to participate}

All procedures performed in the study were in accordance with the ethical standards of the institutional research committee and with the 1964 Helsinki declaration and its later amendments. The study was ethically approved by the research committee and reviewed by the faculty of medicine Cairo University board on 18/7/2017.

\section{Consent for publication}

Not applicable

\section{Competing interests}

The authors declare that they have no competing interests.

Received: 15 December 2018 Accepted: 22 August 2019

Published online: 06 September 2019

\section{References}

1. The national institute of neurological disorders and stroke rt-PA stroke study group. Tissue plasminogen activator for acute ischemic stroke. N Engl J Med. 1995;333:1581-7.

2. Demaerschalk BM, Kleindorfer DO, Adeoye OM, Demchuk AM, Fugate JE, Grotta JC, et al. Scientific statement for inclusion and exclusion criteria for intravenous alteplase in acute ischemic stroke: A statement for healthcare professionals from American heart association/ American stroke association. Stroke. 2016;47:581-641.
3. Brott T, Adams HP Jr, Olinger CP, Marler JR, Barsan WG, Biller J, et al. Measurements of acute cerebral infarction: a clinical examination scale. Stroke. 1989;20:864-70.

4. Bhardwaj A, Sharma G, Raina SK, Sharma A, Angra M. Advanced age and higher National Institutes of Health Stroke Scale Score as predictors of poor outcome in ischemic stroke patients treated with alteplase: a study from a tertiary care centre in rural North-west India. J Neurosci Rural Pract. 2017:8(2):236-40.

5. Spaander FH, Zinkstok SM, Baharoglu IM, H G, A P, Traenka C, et al. Sex differences and functional outcome after intravenous thrombolysis. Stroke. 2017:48:699-703.

6. Demchuk AM, Tanne D, Hill MD, Kasner SE, Hanson S, Grond M, and the Multicentre tPA Stroke Survey Group, et al. Predictors of good outcome after intravenous rt-PA for acute ischemic stroke: The multi-centre tPA acute stroke survey. Neurology. 2001;57:474-80.

7. Vogt G, Laage R, Shuaib A, Schneider A, VISTA Collaboration. Initial lesion volume is an independent predictor of clinical stroke outcome at day 90: an analysis of the Virtual International Stroke Trials Archive (VISTA) database. Stroke. 2012;43:1266-72.

8. Yang Y, Wang A, Zhao X, Wang C, Liu L, Zheng H, et al. The Oxfordshire Community Stroke Project classification system predicts clinical outcomes following intravenous thrombolysis: a prospective cohort study. Ther Clin Risk Manag. 2016;12:1049-56.

9. Fuentes B, Martínez-Sánchez P, Alonso D, Egido J, Reig-Roselló G, Díaz-Otero F, et al. Madrid Stroke Network. Efficacy of intravenous thrombolysis according to stroke subtypes: the Madrid Stroke Network data. Eur J Neurol. 2012;19:1568-74.

10. Mustanoja S, Meretoja A, Putaala J, Viitanen V, Curtze S, Atula S, et al. Helsinki Stroke Thrombolysis Registry Group. Outcome by stroke etiology in patients receiving thrombolytic treatment: descriptive sub-type analysis. Stroke. 2011;42:102-6.

11. Van Swieten JC, Koudstaal PJ, Visser MC, Schouten HJ, van Gijn J. Interobserver agreement for the assessment of handicap in stroke patients. Stroke. 1988;19:604-7.

12. Adams HP Jr, Bendixen BH, Kappelle LJ, Biller J, Love BB, Gordon DL, et al. Classification of subtype of acute ischemic stroke. Definitions for use in a multicenter clinical trial. TOAST:Trial of Org 10172 in Acute Stroke Treatment. Stroke. 1993;24:35-41.

13. Bamford J, Sandercock P, Dennis M, Burn J, Warlow C. Classification and natural history of clinically identifiable subtypes of cerebral infarction. Lancet. 1991;337(8756):1521-6.

14. Chalela JA, Kidwell CS, Nentwich LM, Luby M, Butman JA, Demchuk AM, et al. Magnetic resonance imaging and computed tomography in emergency assessment of patients with suspected acute stroke: a prospective comparison. Lancet. 2007;369:293-8.

15. Mozaffarian D, Benjamin EJ, Go AS, Arnett DK, Blaha MJ, Cushman M, on behalf of the American Heart Association Statistics Committee and Stroke Statistics Subcommittee, et al. Heart disease and stroke statistics update a report from the American Heart Association. Circulation. 2016;133(4):e38-48.

16. Muchada M, Rodriguez-Luna D, Pagola J, Flores A, Sanjuan E, Meler P, et al. Impact of time to treatment on tissue-type plasminogen activator-induced recanalization in acute ischemic stroke. Stroke. 2014;45:2734-8.

\section{Publisher's Note}

Springer Nature remains neutral with regard to jurisdictional claims in published maps and institutional affiliations. 